\title{
Modified pressure plate method for measuring adsorption moisture retention curves
}

\author{
Chi Feng ${ }^{1,2, *}$, and Hans Janssen ${ }^{3}$ \\ ${ }^{1}$ School of Architecture and Urban Planning, Chongqing University, 400045 Chong Qing, PR China \\ ${ }^{2}$ Key Laboratory of New Technology for Construction of Cities in Mountain Area, 400045 Chong Qing, PR China \\ ${ }^{3}$ KU Leuven, Department of Civil Engineering, Building Physics Section, 3001 Leuven, Belgium
}

\begin{abstract}
The pressure plate is one of the most widely used methods to measure the moisture retention curves of porous materials in the over-hygroscopic range. Due to its working principle and operational protocol, the traditional pressure plate method is only applicable to the desorption process. In this study, we propose a novel method to measure the moisture retention curves for the adsorption process. Only simple modifications to the traditional pressure plate setup are needed, and minor changes in the experimental procedures are required. Validation measurements are performed on three representative porous building materials: calcium silicate, autoclaved aerated concrete and ceramic brick. Results from the modified pressure plate method are compared with those from the semi-permeable membrane and the psychrometer methods. The modified pressure plate method successfully provides similar results to the other two established methods for all three materials, and hence proves to be reliable in determining the adsorption moisture retention curves in the over-hygroscopic range.
\end{abstract}

\section{Introduction}

Moisture has a significant impact on buildings and the built environment: not only buildings' energy efficiency and components' service life but also the indoor climate and air quality are closely related to moisture [1-4]. Consequently, it is of great value to have a profound understanding and optimized control of moisture-related processes within building envelopes.

To analyze these processes, the hygric properties of porous building materials are usually indispensable. These properties describe the materials' capability to store and transport moisture. For the storage aspect, the moisture storage functions are often used. In the hygroscopic range where the activity of water vapor is dominant, such functions are called sorption isotherms and are often determined by the desiccator method or the dynamic vapor sorption method $[5,6]$. In the over-hygroscopic range where liquid water plays a more important role, such functions are called moisture retention curves, in most cases measured through the pressure plate method [7].

The pressure plate method was first proposed for soil science in 1948 by Richards [8] and then became widely adopted. Nowadays it has been standardized for not only soil (e.g. by the ISO 11274 standard [9]) but also other porous media, including porous building materials (e.g. by the ASTM C1699 standard [10]). During a typical measurement, compressed air is applied into a pressure vessel for pressure regulation, and a piece of porous ceramic plate is used as the axis translation medium. The water in the samples is then expelled at the exerted pressure and the moisture content is determined after reaching equilibrium. Unfortunately, unlike the desiccator test, the pressure plate test is only applicable to the desorption process but incapable of the adsorption process - which is equally important.

Recently, Fredriksson and Johansson [11] modified the traditional pressure plate and applied it to the adsorption process of brick and spruce. Their results 
seemed promising. However, their setup is very complicated (Fig.1) and only applies to the capillary pressure $\left(p_{c}, \mathrm{~Pa}\right)$ range from 0 to $-5 \cdot 10^{5} \mathrm{~Pa}$. To overcome these drawbacks, we propose a differently modified pressure plate for the adsorption process (Fig.2). The equipment setup and operational procedures are only slightly changed from the traditional version, and the applicable $p_{c}$ range remains very much the same.

In the following sections, we first explain our modifications to the pressure plate. After that, validation measurements are performed and the results are confronted against the data obtained from other established methods, before the final conclusions are drawn.

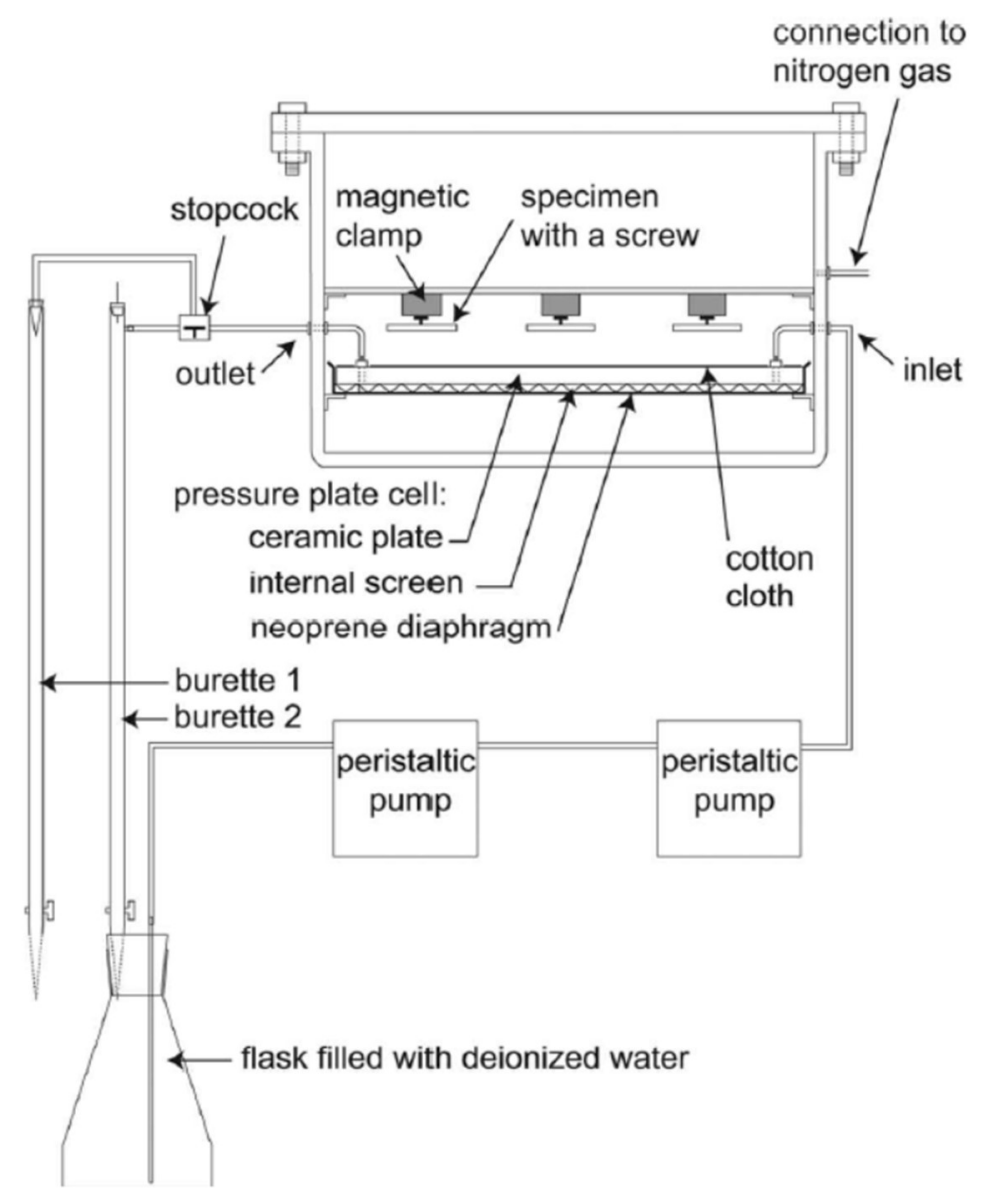


Fig.1. The adsorption pressure plate modified by Fredriksson and Johansson [11]

Fig.2. A schematic of the adsorption pressure plate proposed in this study

Table 1. Fundamental properties of target materials $\left(21-23^{\circ} \mathrm{C}\right)$ [12]

\begin{tabular}{cccccccc}
\hline Material & $\rho_{\text {bulk }}\left(\mathrm{kg} \cdot \mathrm{m}^{-3}\right)$ & $\phi(\%)$ & ${ }^{\mathrm{a}}$ & $u_{84.7 \%^{\mathrm{b}}\left(\mathrm{kg} \cdot \mathrm{kg}^{-1}, \%\right)}$ & $r_{50}(\mathrm{~m})$ & $A_{\text {cap }}{ }^{\mathrm{c}}\left(\mathrm{kg} \cdot \mathrm{m}^{-2} \mathrm{~s}^{-0.5}\right)$ & $w_{\text {cap }}\left(\mathrm{kg} \cdot \mathrm{m}^{-3}\right)$ \\
\hline CS & 271 & 89.1 & 2.0 & 2.9 & $3.2 \times 10^{-7}$ & 1.01 & 756 \\
AAC & 462 & 81.4 & 7.4 & 3.1 & $-\mathrm{d}$ & 0.046 & 312 \\
CB & 1818 & 32.6 & 11.6 & $<0.05^{\mathrm{d}}$ & $4.1 \times 10^{-6}$ & 0.61 & 210 \\
\hline
\end{tabular}

${ }^{\mathrm{a}}$ Determined from dry cup test (RH $\left.11.3 \% \sim 53.5 \%\right)$;

${ }^{\mathrm{b}}$ Adsorption from the dry state;

${ }^{c}$ Values for $20^{\circ} \mathrm{C}$;

${ }^{\mathrm{d}}$ Cannot be determined accurately.

\section{Materials and methods}

\subsection{Materials}

For the validation of our proposed adsorption pressure plate, we conduct measurements on three frequently studied porous building materials - calcium silicate (CS), autoclaved aerated concrete (AAC) and ceramic brick (CB). For a better understanding, their fundamental properties are summarized in Table 1[12], including the bulk density $\left(\rho\right.$ bulk, $\left.\mathrm{kg} \cdot \mathrm{m}^{-3}\right)$, the open porosity $(\phi)$, the vapor diffusion resistance factor ( ), the equilibrium moisture content at $\mathrm{RH} 84.7 \%\left(u_{84.7 \%}, \mathrm{~kg} \cdot \mathrm{kg}^{-1}\right)$, the median pore-throat radius $\left(r_{50}, \mathrm{~m}\right)$, the capillary of $0.5 \mathrm{~cm}$. Their dry mass (and later the wet mass) is measured after drying in a ventilated oven at $70^{\circ} \mathrm{C}$ and cooling down to $23 \pm 0.5^{\circ} \mathrm{C}$ (the temperature for all measurements), by an electronic balance reading $1 \mathrm{mg}$. For each material, four duplicate samples are tested.

During the test, the porous ceramic plate is pre-saturated and placed in the pressure vessel as usual. However, on the plate a piece of moderately moist filter paper is laid directly, without the kaolin layer always used in the traditional pressure plate test. Above the filter paper lie the (relatively) dry samples for the test (Fig.3 a). Next, the pressure vessel is sealed as normal but the outlet is kept in direct contact with pure water (Fig.3 b), unlike in the traditional setup where a burette is used to

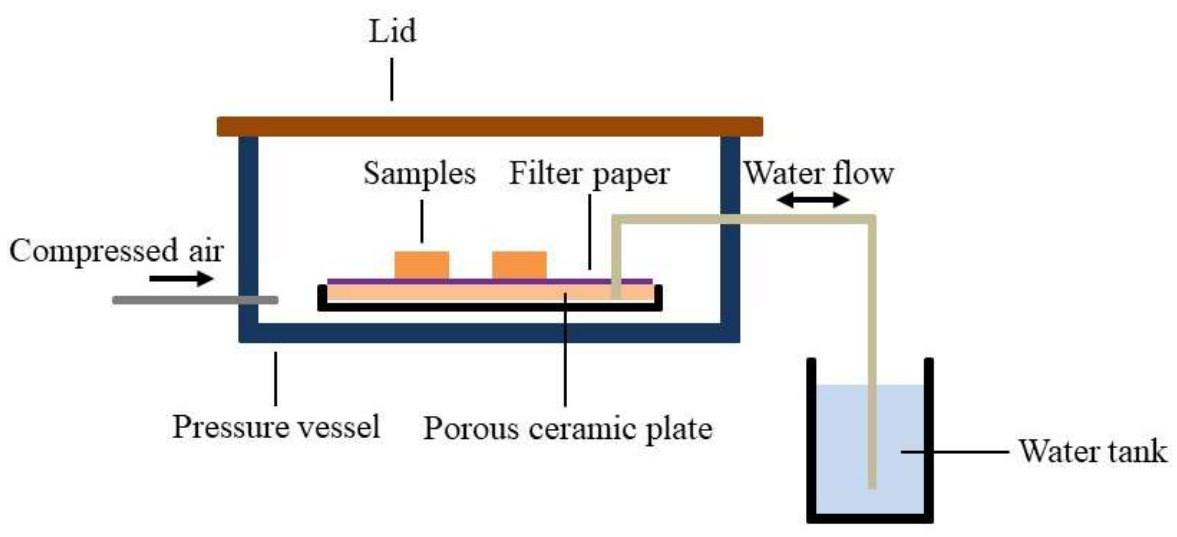

absorption coefficient $\left(A_{c a p}, \mathrm{~kg} \cdot \mathrm{m}^{-2} \mathrm{~s}^{-0.5}\right)$, and the capillary moisture content $\left(w_{\text {cap }}, \mathrm{kg} \cdot \mathrm{m}^{-3}\right)$. These three materials vary in hygroscopicity and capillarity, representing a spectrum of porous building materials with respect to the hygric characteristics, and are therefore often chosen as target materials $[13,14]$.

\subsection{Methods}

For the adsorption pressure plate test, raw materials are cut into samples with a diameter of $5 \mathrm{~cm}$ and a thickness measure the water outflow. After that compressed air is supplied into the vessel initially at a pressure roughly $2 \cdot 10^{5} \mathrm{~Pa}$ higher than the target value. At this stage, the water in the filter paper and in the porous ceramic plate slowly flows out of the vessel into the container with pure water, creating an uninterrupted hydraulic contact in the system. After 1 2 hours, when the pressure equilibrium has been realized, the air pressure in the vessel is slowly decreased to the target value, causing a suction from the water outlet into the pressure vessel. 
Now the samples can absorb moisture at this target pressure while the suction ensures a continuous water supply from the water tank. After reaching equilibrium,

a. Samples in the pressure vessel the moisture content of samples can be determined gravimetrically. Throughout the test, the air pressure fluctuation is within $0.1 \%$, causing negligible impact.

b. Overview of the setup

Fig.3. Photos of the adsorption pressure plate proposed in this study

\section{Results and discussions}

Fig.4 illustrates the results for the three target materials obtained from the adsorption pressure plate proposed in this study, in the $p_{c}$ range between $-1 \cdot 10^{5}$ and $-1.1 \cdot 10^{6} \mathrm{~Pa}$. For comparison, the adsorption data for the same materials measured by the semi-permeable membrane test and the psychrometer test [14] - two established methods - are also included.

As is clearly shown, for all three target materials, the results obtained from our adsorption pressure plate agree reasonably well with the results from the other two methods. For the $p_{c}$ range close to $0 \mathrm{~Pa}$, all three methods exhibit large scatters, meaning that none of them is perfectly reliable here, calling for further investigations. For the lower $p_{c}$ values, the adsorption

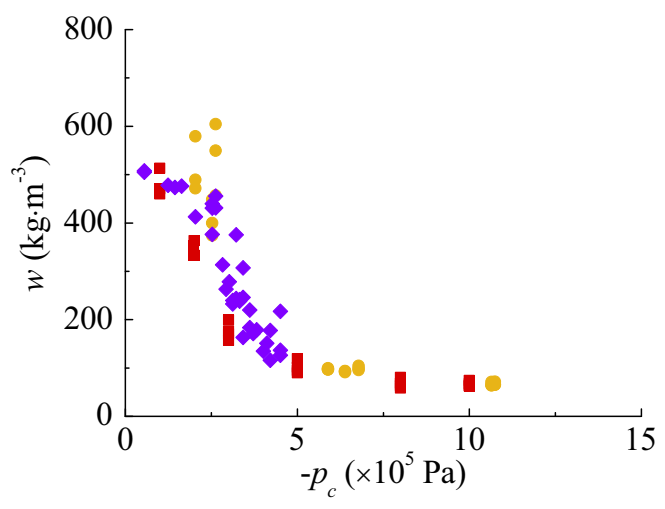

a) $\mathrm{CS}$

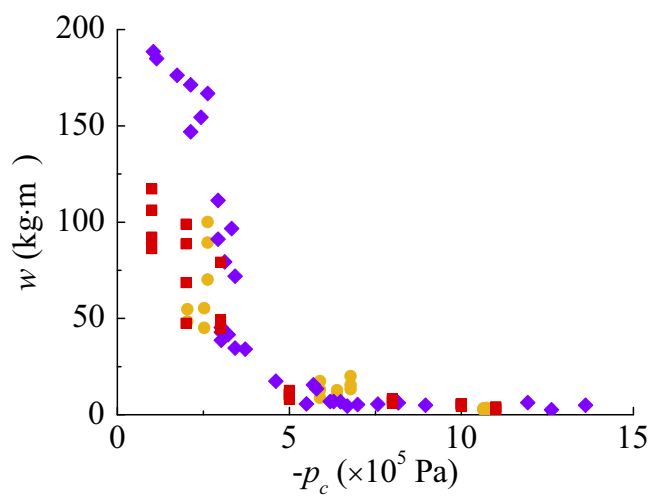

c) $\mathrm{CB}$ pressure plate shows some minor underestimations occasionally, most probably due to the fact that the equilibrium has not been fully reached for these cases. But in general, the adsorption pressure plate does a good job.

It should be noted that without a burette it is difficult to determine the equilibrium state during the test. Thus, the adsorption is interrupted from time to time to check the moisture content of the samples. The time needed for reaching equilibrium differs by different materials, but in most cases 2 3 weeks seem to suffice. In the future, we will make further modifications to the setup by integrating a burette into the water supply system, so as to facilitate the observation on equilibrium.

It should also be mentioned that when the samples are laid on the filter paper before the compressed air is

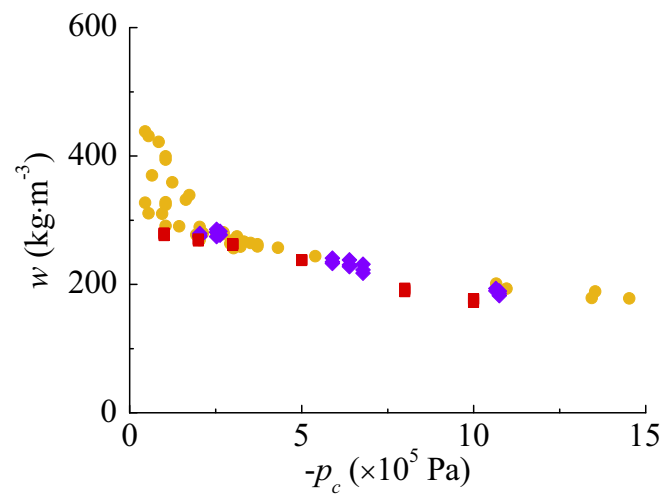

b) $\mathrm{AAC}$

- Adsorption pressure plate

- Semi-permeable membrane

- Psychrometer

Fig.4. Results obtained from the adsorption pressure plate and another two methods 
supplied, it is possible for them to absorb moisture from the moist filter paper and the pre-saturated porous ceramic plate freely. That's the reason why the filter paper should only be moderately moist and the compressed air should be exerted as soon as possible, in order to avoid the situation that samples quickly reach a

\section{Conclusions}

In this study, the equipment setup and the operational procedures of the traditional pressure plate are modified, to perform adsorption tests in the over-hygroscopic range. Three typical porous building materials - calcium silicate, autoclaved aerated concrete and ceramic brick are tested for validation. Results obtained from the proposed adsorption pressure plate are compared with the data from the semi-permeable membrane and the psychrometer tests. The reliability of the adsorption pressure plate has been proved, albeit some minor deviations in the low $p_{c}$ range and relatively large scatters in the high $p_{c}$ range.

This project is supported by EU H2020 project "RI Build Robust Internal Thermal Insulation of Historic Buildings" (project No. 637268).

\section{References}

1. H. Zhang, H. Yoshino, K. Hasegawa, J. Liu, W. Zhang, H. Xuan, Energy Build. 139, 214-223 (2017)

2. M. D'Orazio, G. Maracchini, Energy Build. 204, 109500 (2019)

3. T. Yan, Z. Sun, X. Xu, H. Wan, G. Huang, Energy. 183, 1278-1294 (2019) moisture content higher than the value corresponding to the aimed $p_{c}$. The ceramic plate typically has very fine pores though, so without external air/suction pressure the moisture transport between the samples and the plate would be negligibly slow still.

4. C. Feng, S. Roels, H. Janssen, Build. Environ. 164, 106343 (2019)

5. C. Feng, H. Janssen, C. Wu, Y. Feng, Q. Meng, Build. Environ. 69, 64-71 (2013)

6. B. Rudy, L. Matthieu, A. Jean-Emmanuel, Constr. Build. Mater. 141, 140-151 (2017)

7. M. Bittelli, M. Flury, Soil Sci. Soc. Am. J. 73, 1453-1460 (2009)

8. L. Richards, Soil Sci. 66, 105-110 (1948)

9. ISO 11274: 2019 (E) Soil quality - Determination of the water-retention characteristic - Laboratory methods (2019)

10. ASTM C1699 - 09 (Reapproved 2015): Standard Test Method for Moisture Retention Curves of Porous Building Materials Using Pressure Plates (2015)

11. M. Fredriksson, P. Johansson, Dry. Technol. 34, 132-141 (2016)

12. C. Feng, H. Janssen, Build. Environ. 134, 21-34 (2018)

13. S. Roels, J. Carmeliet, H. Hens, et al. HAMSTAD Work Package 1: Final Report - Moisture Transfer Properties and Materials Characterisation, EU Contract GRD1-1999-20007 (2003)

14. C. Feng, H. Janssen, Build. Environ. 152, 39-49 (2019) 